\title{
Equilibrium storage with multiple commodities
}

\author{
Kazuo Nishimura, John Stachurski* \\ Institute of Economic Research, Kyoto University, Yoshida honmachi, Sakyo-ku, Kyoto 606-8501, Japan
}

\section{A R T I C L E I N F O}

\section{Article history:}

Received 27 October 2007

Received in revised form 23 June 2008

Accepted 3 July 2008

Available online 25 July 2008

\section{JEL classification:}

C61

C62

Keywords:

Commodities

Dynamic programming

Stability

\section{A B S T R A C T}

This paper introduces a multisector model of commodity markets with storage, where equilibrium is defined by profit maximization, arbitrage and market clearing conditions. We then solve for the decentralized equilibrium via a corresponding dynamic program. We also describe the dynamics of the model, establishing geometric ergodicity, a Law of Large Numbers and a Central Limit Theorem.

(c) 2008 Elsevier B.V. All rights reserved.

\section{Introduction}

Given their volatility and importance to politically sensitive coalitions, markets for primary commodities have often attracted intervention by governments aimed at stabilizing producer incomes. Ideally, manipulation of endogenously determined variables requires careful analysis of the equilibrium process. For this and other reasons economists have been motivated to construct models which replicate the key features of primary commodity markets.

Perhaps the most successful attempt to frame such a model is found in the seminal work of Samuelson (1971) and Schectman and Scheinkman (1983). These analyses combine profit maximization, arbitrage conditions and market clearing to derive a complete system of equilibrium prices and quantities over time for a single market which features stochastic supply and speculative investment. ${ }^{1}$

Given their simple arbitrage-based restrictions and endogenous determination of consumption, speculative investment and price, these commodity pricing models provide an attractive framework for empirical analysis. They have formed the basis of numerous quantitative studies, including Deaton and Laroque (1992, 1996), Chambers and Bailey (1996) and Ng and Ruge-Murcia (2000).

This paper introduces a multisector model of commodity prices in the spirit of Samuelson (1971) and Schectman and Scheinkman (1983). Our motivation is to provide the theoretical foundations for models which replicate commodity markets more closely by taking into account the joint determination of prices and quantities across related commodities. A multisector approach can accommodate the impact of demand and supply conditions for one commodity on the price and quantity observed in the market for another.

\footnotetext{
* Corresponding author.

E-mail addresses: nishimura@keir.kyoto-u.ac.jp (K. Nishimura), john@keir.kyoto-u.ac.jp (J. Stachurski).

1 Numerous other authors have contributed to the theory of the one-sector commodity pricing model. See, for example, Williams and Wright (1991) or Routledge et al. (2000).
} 


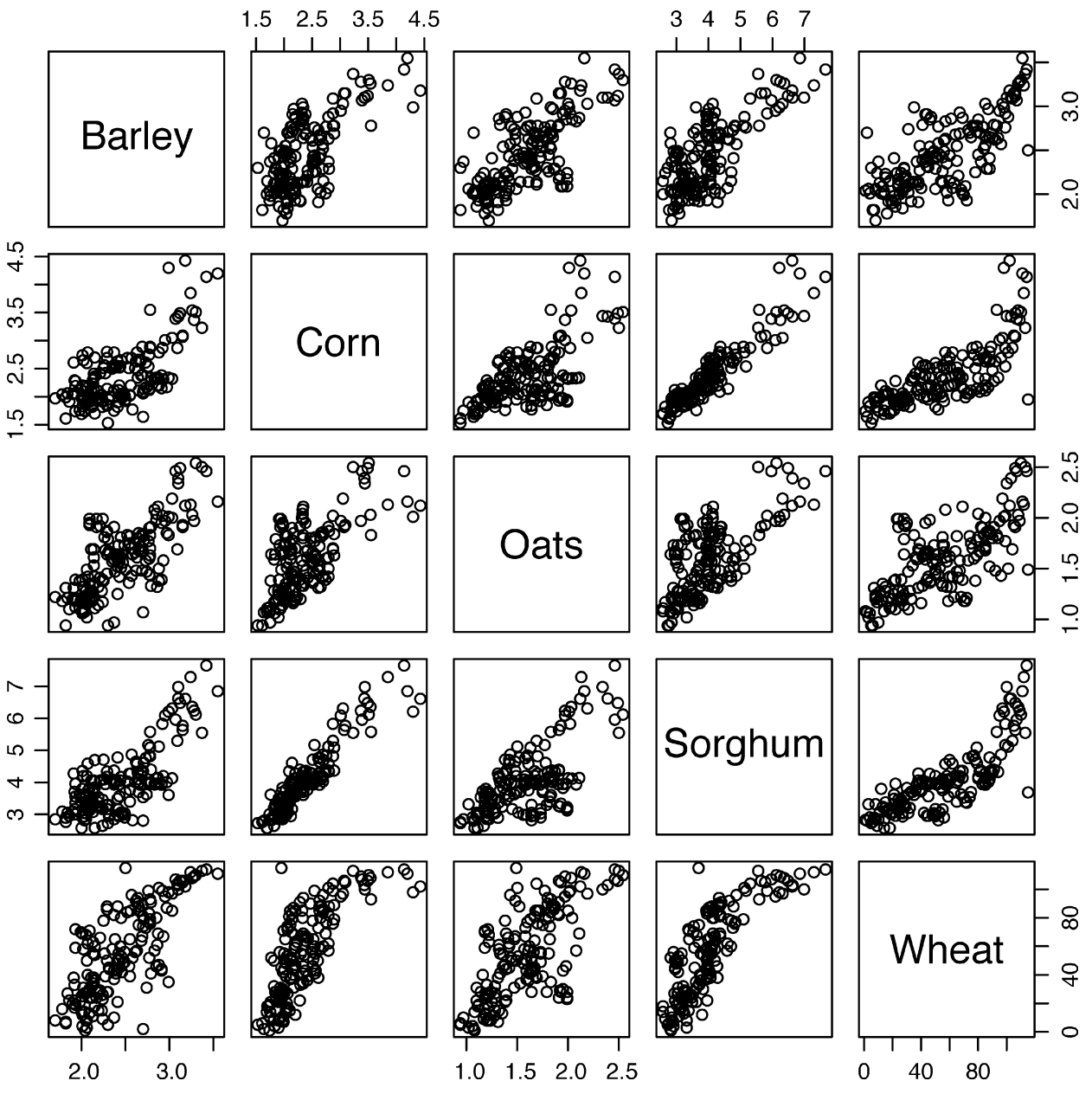

Fig. 1. Price correlations, grains.

In general, prices and quantities of related commodities are jointly determined because of contemporaneously correlated shocks on the supply side and substitutability or complementarity on the demand side. For example, the markets for grains such as corn, sorghum, oats, wheat and barley have traditionally been closely integrated, as suggested by the scatter plot matrix for prices over the period from January 1994 to July 2007 shown in Fig. 1. ${ }^{2}$ Altered supply or demand conditions for one grain impact on prices for other grains, in this case due mainly to substitutability of feed grains (as a function of energy content). ${ }^{3}$

The fact that a multisector equilibrium commodity pricing model has not been developed to date appears largely due to technical difficulties. While the one-sector model has proven to be highly tractable, the multisector model is considerably less so. For example, in the one-sector model interiority and first order conditions are straightforward, investment is monotone in quantity, and the state variable (supply) follows a renewal process which guarantees stationarity and ergodicity. In the multisector case none of these features remain, and analysis is correspondingly more difficult.

In this paper we show that despite these difficulties a multisector model can be successfully developed and analyzed. We frame a market with $M$ commodities and define equilibrium prices and quantities via profit maximization, arbitrage and market clearing conditions. We then introduce a planner's problem, the first order conditions for which yield the arbitrage conditions of the decentralized market. The decentralized equilibrium can then be computed via dynamic programming techniques.

\footnotetext{
2 The data are monthly average prices in the US over the stated period. Source: Agriculture Statistics Board.

3 It has also been argued that the prices of seemingly unrelated commodities are correlated even after controlling for relevant macroeconomic variables (Pindyck and Rotemburg, 1990). Our model is not suitable for addressing this issue as we envisage relationships between the commodities on either the demand or supply side. Without such relationships our $M$-sector model reduces to $M$ decoupled one-sector models.
} 
In the dynamic program, we have treated an unbounded reward problem via weighted supremum norms. ${ }^{4}$ Thus we require neither bounded rewards - which excludes common parametric formulations - nor bounded shocks. Bounded shocks are a standard technique used to compactify the state space and thereby bound rewards, but have the disadvantage of excluding many shock distributions used routinely in econometrics. We avoid placing additional assumptions on rewards and shocks by careful choice of the weighting function. (For example, we do not require the homogeneity conditions used in Boyd, 1990; Alvarez and Stokey, 1998.)

Our other results concern dynamics of the state process. Establishing global stability (ergodicity) is considerably more complicated than in the one-sector model, where policies are monotone and the state follows a renewal process. ${ }^{5}$ Using an alternative approach, we are able to provide simple conditions under which the equilibrium process for the stock is asymptotically stationary and geometrically ergodic.

Geometric ergodicity in turn leads to characterization of the sample paths, which are shown to satisfy a Strong Law of Large Numbers and a Central Limit Theorem. These properties open up a range of consistent estimation techniques (cf., e.g., the simulation-based methods of Duffie and Singleton, 1993).

\subsection{Outline}

The next section introduces the multisector model and defines competitive equilibrium. Section 3 sets up a corresponding dynamic program, and establishes the connection between this programming problem and the decentralized equilibrium. Section 4 considers dynamics under the equilibrium. Section 5 concludes, and any remaining proofs are given in Appendix A.

\section{Speculative prices}

In this section we construct a multisector version of Samuelson's commodity pricing model. In subsequent sections we show that equilibrium storage by speculators in the decentralized market is equal to the optimal investment policy of a planner maximizing an discounted revenue stream for the final producers.

In what follows, $\mathbb{R}_{+}^{M}:=[0, \infty)^{M}$ and $\mathbb{R}_{++}^{M}:=(0, \infty)^{M}$. For $x$ and $y$ in $\mathbb{R}^{M}$ the relation $x \leq y$ means that $y-x \in \mathbb{R}_{+}^{M}$, while $x \ll y$ means that $y-x \in \mathbb{R}_{++}^{M}$. The notation $[x, y]$ denotes an order interval: $[x, y]$ is all $z \in \mathbb{R}^{M}$ such that $x \leq z \leq y$; $(x, y)$ is all $z$ with $x \ll z \ll y$, and so on. Let $\partial \mathbb{R}_{+}^{M}$ be the boundary $\mathbb{R}_{+}^{M} \backslash \mathbb{R}_{++}^{M}$.

The inner product of $x$ and $y$ is denoted $\langle x, y\rangle$, and $\|x\|:=\langle x, x\rangle^{1 / 2}$ is the Euclidean norm. For $g: \mathbb{R}^{M} \rightarrow \mathbb{R}$, the symbol $\nabla g$ denotes the vector of partial derivatives when it exists, and $D_{h} g(x)$ is the directional derivative of $g$ at $x$ in the direction $h$ :

$$
D_{h} g(x):=\lim _{\theta \downarrow 0} \frac{g(x+\theta h)-g(x)}{\theta}
$$

We use $\lambda$ to denote Lebesgue measure on $\mathbb{R}_{+}^{M}$, while $\mathcal{B}\left(\mathbb{R}_{+}^{M}\right)$ is the Borel sets and $L_{1}\left(\mathbb{R}_{+}^{M}\right)$ is the Lebesgue integrable functions. A distribution is a Borel probability measure on $\mathbb{R}_{+}^{M}$.

The market has $M$ commodities, the vector of spot prices for which is given at time $t$ by $p_{t}=\left(p_{t}^{m}\right)_{m=1}^{M} \in \mathbb{R}_{+}^{M}$. Demand for the commodities comes from firms (final producers), who use the commodities as inputs to their production process, and from speculators, who purchase the commodities for future sale. Let the risk-free interest rate $r$ be constant, and set $\rho:=(1+r)^{-1}$.

The firms demand a vector $C_{t}$ of the commodities according to the profit maximization problem:

$$
\max _{C_{t}} \Pi_{t}\left(C_{t}\right), \quad \Pi_{t}(x):=F(x)-\left\langle p_{t}, x\right\rangle
$$

where $F(x)$ is the output of the final good given input vector $x$, and the price of the final good has been normalized to one.

Assumption 2.1. The function $F: \mathbb{R}_{+}^{M} \rightarrow \mathbb{R}_{+}$is strictly concave, strictly increasing, continuous and differentiable on $\mathbb{R}_{++}^{M}{ }^{6}$

In addition, for our analysis to succeed we require that equilibrium demand is strictly positive in each period. In the multisector case obtaining conditions on the primitives of the model under which this property holds is a nontrivial problem. We show that the following condition is sufficient.

Assumption 2.2. For any $x$ on the boundary of $\mathbb{R}_{+}^{M}$ and any vector $h$ which points to the interior, the directional derivative of $F$ at $x$ in the direction $h$ is infinite. That is, $D_{h} F(x)=\infty$ for all $x \in \partial \mathbb{R}_{+}^{M}$ and $h \in \mathbb{R}_{+}^{M}$ such that $x+h \in \mathbb{R}_{++}^{M}$.

\footnotetext{
4 See Hernández-Lerma and Lasserre (1999, Chapter 8) for an excellent discussion of the theory of dynamic programming with weighted sup-norms. For an alternative to the weighted sup-norm approach, see Le Van and Morhaim (2002) or Rincon-Zapatero and Rodriguez-Palmero (2003).

5 The dynamics of the single sector commodity pricing model were investigated in detail by Schectman and Scheinkman (1983). Confirming a conjecture of Samuelson (1971), they show that the process for the state (the stock of the commodity) converges asymptotically to a unique stationary distribution. Bobenrieth et al. (2002) established geometric ergodicity and investigated other properties of the stationary distribution.

6 Strict concavity is assumed because other inputs such as labor and capital are held fixed, in which case constant returns to scale in all inputs requires decreasing returns to scale in the commodity vector alone.
} 
Example 2.1. The Cobb-Douglas technology $F(x)=\prod_{m=1}^{M}\left(x^{m}\right)^{a_{m}}$ satisfies all of the conditions of Assumptions 2.1 and 2.2 when $a:=\sum_{m=1}^{M} a_{m}<1$. The only element of this claim which requires proof is the interiority condition in Assumption 2.2 . For the proof, pick any $x \in \partial \mathbb{R}_{+}^{M}$ and any $h \in \mathbb{R}^{M}$ with $y:=\chi+h \in \mathbb{R}_{++}^{M}$. We have

$$
\begin{aligned}
& \theta^{a} F(y)=F(\theta y) \leq F((1-\theta) x+\theta y)=F(x+\theta h) \\
& \therefore \quad \theta^{a-1} F(y) \leq \frac{F(x+\theta h)-F(x)}{\theta} \quad(\because F(x)=0)
\end{aligned}
$$

Since $y \gg 0$ we have $F(y)>0$, and as $a<1$ the left-hand side converges to infinity when $\theta \downarrow 0$.

Aside from firms, there exists a unit mass of identical speculators who are able to store the commodities between periods. Purchasing $I^{m}$ units of good $m$ yields $\alpha^{m} I^{m}$ units next period, where $\alpha^{m} \in(0,1)$ parameterizes storage cost, or depreciation. Hence $I_{t}=\left(I_{t}^{m}\right)_{m=1}^{M} \in \mathbb{R}_{+}^{M}$ carried over from time $t$ yields $\Lambda I_{t}$ at $t+1$, where

$$
\Lambda:=\operatorname{diag}\left(\alpha^{1}, \ldots, \alpha^{M}\right)=\left(\begin{array}{ccc}
\alpha^{1} & \cdots & 0 \\
\vdots & \ddots & \vdots \\
0 & \cdots & \alpha^{M}
\end{array}\right) \quad \text { and } \alpha^{m} \in(0,1)
$$

Aggregate supply $X_{t}$ in the market at time $t$ is the sum of $\Lambda I_{t-1}$ and a "harvest" $W_{t}$ :

$$
X_{t}=\Lambda I_{t-1}+W_{t}
$$

Regarding the harvest process we make the following assumption:

Assumption 2.3. The shocks $\left(W_{t}\right)_{t \geq 1}$ are independent, identically distributed $\mathbb{R}_{+}^{M}$-valued random vectors with common distribution $\phi$. In addition, $\phi\left(\partial \mathbb{R}_{+}^{M}\right)=0$ and

$$
\mu:=\mathbb{E}\left\|W_{t}\right\|=\int\|z\| \phi(\mathrm{d} z)<\infty
$$

The assumption that $\phi\left(\partial \mathbb{R}_{+}^{M}\right)=0$ implies that $X_{t}$ lies in $\mathbb{R}_{++}^{M}$ for all $t$ with probability one, in which case we need not concern ourselves with infinite prices on the boundary $\partial \mathbb{R}_{+}^{M}$. The sequence of shocks $\left(W_{t}\right)_{t>1}$ is defined on an arbitrary probability space $(\Omega, \mathcal{F}, \mathbb{P}){ }^{7}$ and $\mathbb{E}$ denotes expectation with respect to $\mathbb{P}$.

To construct a competitive equilibrium for the commodity market we consider input demand, investment and pricing functions $c, i$ and $p$ from $\mathbb{R}_{++}^{M} \rightarrow \mathbb{R}_{+}^{M}$. Given $c, i$ and $p$, the process for quantities and prices evolves according to

$$
I_{t}=i\left(X_{t}\right), \quad C_{t}=c\left(X_{t}\right), \quad p_{t}=p\left(X_{t}\right), \quad X_{t+1}=\Lambda I_{t}+W_{t+1}
$$

For this system of functions to be an equilibrium requires market clearing and profit maximization, or

$$
c(x)+i(x)=x \text { and } \nabla F(c(x))=p(x)
$$

for $x \in \mathbb{R}_{++}^{M}$. In addition, we introduce the following arbitrage condition. Given $x \in \mathbb{R}_{+}^{M}$ and investment policy $i$, we say that $h \in \mathbb{R}^{M}$ is a feasible variation at $x$ if $i(x)+h \in[0, x]$. The set of all feasible variations at $x$ is denoted $F_{v}^{i}(x)$. Assuming speculators are risk neutral, nonexistence of arbitrage requires that $i$ and $p$ satisfy

$$
\rho \int\langle p(\Lambda i(x)+z), \Lambda h\rangle \phi(\mathrm{d} z) \leq\langle p(x), h\rangle \quad \forall h \in F_{v}^{i}(x)
$$

for $x \in \mathbb{R}_{++}^{M}$. If the condition (4) fails for some $x$ then a deviation from $i(x)$ in the feasible direction $h$ yields strictly positive expected profits for speculators, and hence is not an equilibrium.

\section{The planner's problem}

In order to construct a set of prices and quantities which satisfy (3) and (4) we introduce a planning problem, the optimal policy for which generates the decentralized equilibrium. The planning problem can be stated as

$$
\max _{i \in \mathcal{I}} \mathbb{E} \sum_{t \geq 0} \rho^{t} F\left(X_{t}-i\left(X_{t}\right)\right)
$$

subject to $X_{t+1}=\Lambda i\left(X_{t}\right)+W_{t+1}, \quad X_{0} \sim \psi_{0}$

where $F, \rho,\left(W_{t}\right)_{t \geq 1}$ and $\Lambda$ are as defined in Section 2, and $\mathcal{I}$ denotes the set of investment policies $i: \mathbb{R}_{+}^{M} \rightarrow \mathbb{R}_{+}^{M}$ that are Borel measurable and satisfy the feasibility constraint $i(x) \leq x$.

\footnotetext{
${ }^{7}$ Thus, $\mathbb{P}\left\{W_{t} \in \cdot\right\}=\mathbb{P} \circ W_{t}^{-1}=\phi$ on $\mathcal{B}\left(\mathbb{R}_{+}^{M}\right)$.
} 


\subsection{Maximization of the surplus}

The control problem (5) and (6) maximizes the firm's discounted revenue stream. ${ }^{8}$ Before demonstrating that the solution to the planner's problem yields the decentralized equilibrium, we provide some intuition as to why maximizing the firm's discounted revenue stream yields the decentralized equilibrium. In particular, we show that (5) and (6) is a natural objective for the planner, as it is equivalent to maximizing total surplus in the market.

Total surplus is the sum of discounted firm profits, payments to the sellers of the commodities and returns to speculative investors:

$$
\mathbb{E}\left\{\sum_{t \geq 0} \rho^{t} \Pi_{t}\left(C_{t}\right)+\sum_{t \geq 0} \rho^{t}\left\langle p_{t}, W_{t}\right\rangle+\sum_{t \geq 1} \rho^{t}\left[\left\langle p_{t}, \Lambda I_{t-1}\right\rangle-\left\langle p_{t-1}, I_{t-1}\right\rangle\right]\right\}
$$

To see that maximization of this surplus subject to the constraints

$$
X_{t+1}=\Lambda I_{t}+W_{t+1}, \quad I_{t}+C_{t} \leq X_{t}, \quad W_{0}:=X_{0}
$$

is equivalent to (5) and (6), note that optimal paths satisfy $I_{t}+C_{t}=X_{t}$ for all $t \geq 0$, from which we obtain $I_{t}+C_{t}=\Lambda I_{t-1}+W_{t}$, and hence

$$
\left\langle p_{t}, I_{t}\right\rangle+\left\langle p_{t}, C_{t}\right\rangle=\left\langle p_{t}, \Lambda I_{t-1}\right\rangle+\left\langle p_{t}, W_{t}\right\rangle
$$

For each $T \in \mathbb{N}$ the sum $\sum_{t=1}^{T} \rho^{t}\left[\left\langle p_{t}, \Lambda I_{t-1}\right\rangle-\left\langle p_{t-1}, I_{t-1}\right\rangle\right]$ becomes

$$
\sum_{t=1}^{T} \rho^{t}\left[\left\langle p_{t}, I_{t}\right\rangle+\left\langle p_{t}, C_{t}\right\rangle-\left\langle p_{t}, W_{t}\right\rangle-\left\langle p_{t-1}, I_{t-1}\right\rangle\right]=\sum_{t=1}^{T} \rho^{t}\left[\left\langle p_{t}, C_{t}\right\rangle-\left\langle p_{t}, W_{t}\right\rangle\right]+\rho^{T}\left\langle p_{T}, I_{T}\right\rangle-\left\langle p_{0}, I_{0}\right\rangle
$$

Returning to our total surplus, then, for each $T \in \mathbb{N}$ the sum is

$$
\sum_{t=0}^{T} \rho^{t} \Pi_{t}\left(C_{t}\right)+\sum_{t=0}^{T} \rho^{t}\left\langle p_{t}, W_{t}\right\rangle+\sum_{t=1}^{T} \rho^{t}\left[\left\langle p_{t}, \Lambda I_{t-1}\right\rangle-\left\langle p_{t-1}, I_{t-1}\right\rangle\right]=\sum_{t=0}^{T} \rho^{t} F\left(C_{t}\right)-\left\langle p_{0}, C_{0}\right\rangle+\left\langle p_{0}, W_{0}\right\rangle+\rho^{T}\left\langle p_{T}, I_{T}\right\rangle-\left\langle p_{0}, I_{0}\right\rangle
$$

Since $X_{0}=W_{0}$ we have $C_{0}+I_{0}=W_{0}$, and hence

$$
\sum_{t=0}^{T} \rho^{t} \Pi_{t}\left(C_{t}\right)+\sum_{t=0}^{T} \rho^{t}\left\langle p_{t}, W_{t}\right\rangle+\sum_{t=1}^{T} \rho^{t}\left[\left\langle p_{t}, \Lambda I_{t-1}\right\rangle-\left\langle p_{t-1}, I_{t-1}\right\rangle\right]=\sum_{t=0}^{T} \rho^{t} F\left(C_{t}\right)+\rho^{T}\left\langle p_{T}, I_{T}\right\rangle
$$

Taking the limit with respect to $T$, this becomes $\sum_{t>0} \rho^{t} F\left(C_{t}\right)$. Hence the surplus maximization problem reduces to (5).

\subsection{Solving the planning problem}

Let $v$ be the value function associated with (5) and (6). That is,

$$
v(x):=\sup _{i \in \mathcal{I}} v_{i}(x), \quad \text { where } v_{i}(x):=\mathbb{E} \sum_{t \geq 0} \rho^{t} F\left(X_{t}-i\left(X_{t}\right)\right)
$$

Here $\left(X_{t}\right)_{t \geq 0}$ obeys the recursion in (6), but with $X_{0}=x$. We call $i \in \mathcal{I}$ optimal if it attains the supremum in (7) for every $x \in \mathbb{R}_{+}^{M}$.

Under our assumptions the value function $v$ is potentially unbounded on $\mathbb{R}_{+}^{M}$, and classical dynamic programming arguments do not apply. We use a weighted norm approach to characterize the optimal policy. Given fixed $\delta \in(\rho, 1)$ and the $\mathbb{R}_{+}^{M}$-valued process

$$
Y_{t+1}=\Lambda Y_{t}+W_{t+1}, \quad Y_{0}=x
$$

we define the weighting function $\kappa: \mathbb{R}_{+}^{M} \rightarrow \mathbb{R}$ by the infinite sum

$$
\kappa(x):=1+\sum_{t=0}^{\infty} \delta^{t} \mathbb{E} F\left(Y_{t}\right), \quad x \in \mathbb{R}_{+}^{M}
$$

Lemma 3.1. The function $\kappa$ is finite, increasing and continuous everywhere on $\mathbb{R}_{+}^{M}$. For any $i \in \mathcal{I}$ we have $v_{i} \leq \kappa$ on $\mathbb{R}_{+}^{M}$.

\footnotetext{
8 Recall that the price of the final commodity is normalized to one.
} 


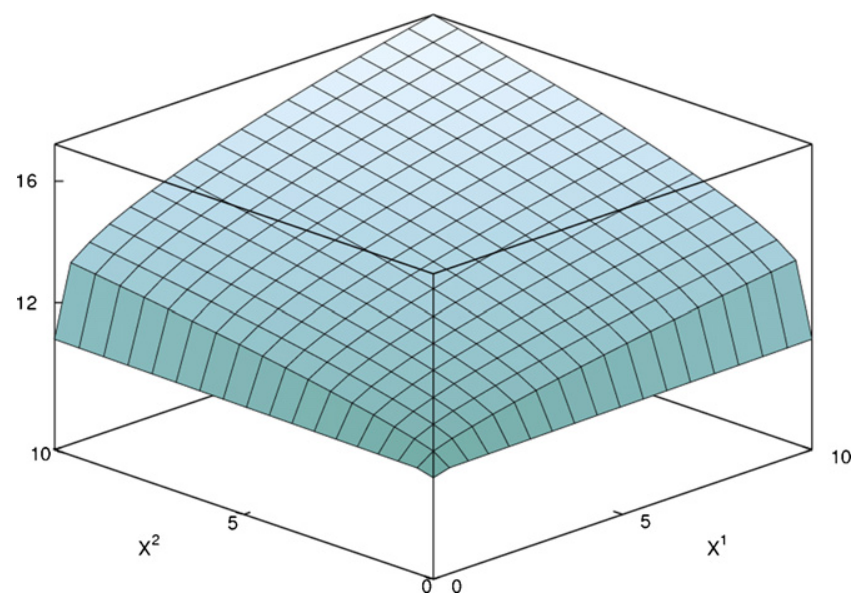

Fig. 2. Value function.

Hence $v$ is finite and $v / \kappa \leq 1$. Define $w: \mathbb{R}_{+}^{M} \rightarrow \mathbb{R}$ to be $\kappa$-bounded if $w / \kappa$ is bounded; that is, if

$$
\|w\|_{\kappa}:=\|w / \kappa\|_{\infty}:=\sup _{x \in \mathbb{R}_{+}^{M}}|w(x) / \kappa(x)|<\infty
$$

The function $w \mapsto\|w\|_{\kappa}$ is a norm on the set of all $\kappa$-bounded functions on $\mathbb{R}_{+}^{M}$. Define $b_{\kappa} \mathcal{B} \mathbb{R}_{+}^{M}$ to be the set of $\kappa$-bounded Borel measurable function on $\mathbb{R}_{+}^{M}$, and $b_{\kappa} c \mathbb{R}_{+}^{M}$ to be those functions which are in addition continuous. The collection of functions $b_{\kappa} \mathcal{B} \mathbb{R}_{+}^{M}$ endowed with the norm $\|\cdot\|_{\kappa}$ is a Banach space. Using continuity of $\kappa$, it can be shown that $b_{\kappa} c \mathbb{R}_{+}^{M}$ is a $\|\cdot\|_{\kappa}$-closed subset of $b_{\kappa} \mathcal{B} \mathbb{R}_{+}^{M}$.

The Bellman operator $T: b_{\kappa} \mathcal{B} \mathbb{R}_{+}^{M} \rightarrow b_{\kappa} \mathcal{B} \mathbb{R}_{+}^{M}$ is defined by

$$
T w(x)=\sup _{0 \leq \xi \leq x}\left\{F(x-\xi)+\rho \int w(\Lambda \xi+z) \phi(\mathrm{d} z)\right\}, \quad x \in \mathbb{R}_{+}^{M}
$$

The operator $T$ is a contraction of modulus $\gamma:=\rho / \delta<1$ on $b_{\kappa} \mathcal{B} \mathbb{R}_{+}^{M}$ with respect to the $\|\cdot\|_{\kappa}$-norm:

Proposition 3.1. $T$ is a well-defined map from $b_{\kappa} \mathcal{B R}_{+}^{M}$ to itself, and

$$
\|T w-T u\|_{\kappa} \leq \gamma\|w-u\|_{\kappa}, \quad w, u \in b_{\kappa} \mathcal{B R}_{+}^{M}
$$

If $w$ is continuous then so is $T w$, and hence $T$ sends $b_{\kappa} c \mathbb{R}_{+}^{M}$ into itself.

We can now characterize the value function and the unique optimal policy in the following way.

Theorem 3.1. The value function $v$ is the unique fixed point of $T$ in $b_{\kappa} \mathcal{B} \mathbb{R}_{+}^{M}$, and for each $w \in b_{\kappa} \mathcal{B} \mathbb{R}_{+}^{M}$ we have $\left\|T^{n} w-v\right\|_{\kappa} \rightarrow 0$ as $n \rightarrow \infty$. In addition, $v$ is continuous, strictly increasing and strictly concave on $\mathbb{R}_{+}^{M}$. A unique optimal policy I $\in \mathcal{I}$ exists. It is continuous, and satisfies

$$
I(x)=\underset{0 \leq \xi \leq x}{\operatorname{argmax}}\left\{F(x-\xi)+\rho \int v(\Lambda \xi+z) \phi(\mathrm{d} z)\right\}, \quad x \in \mathbb{R}_{+}^{M}
$$

Fig. 2 shows (an approximation to) the value function for the two commodity case, where $\alpha^{1}=\alpha^{2}=\rho=0.9, F(x, y)=$ $x^{0.4} y^{0.4}$ and $W=\left(e^{\xi}, e^{\eta}\right)$ with $(\xi, \eta)$ independent standard normal. The approximation was carried out by iterating the Bellman operator, starting at $F \in b_{\kappa} c \mathbb{R}_{+}^{M}$. The sequence converges to $v$ at rate $O\left(\gamma^{n}\right) .{ }^{9}$ Fig. 3 gives the corresponding investment functions $I^{1}\left(x^{1}, x^{2}\right)$ and $I^{2}\left(x^{1}, x^{2}\right)$ (at top and bottom respectively), obtained by solving (10) with $v$ the approximate value function given in Fig. 2.

We also have the following envelope condition:

Proposition 3.2. If $x \gg 0$ then $x-I(x) \gg 0$. In addition, the value function $v$ is differentiable on $\mathbb{R}_{++}^{M}$ and

$$
\nabla v(x)=\nabla F(x-I(x)), \quad x \in \mathbb{R}_{++}^{M}
$$

\footnotetext{
${ }^{9}$ Each iterate was approximated using a continuous piecewise affine function constructed as the infimum of 324 supporting hyperplanes. This technique is related to the method proposed by Santos and Vigo (1998), who suggest approximating value functions by continuous piecewise affine interpolation. (Our algorithm leads to a convex approximation from above, rather than below.)
} 

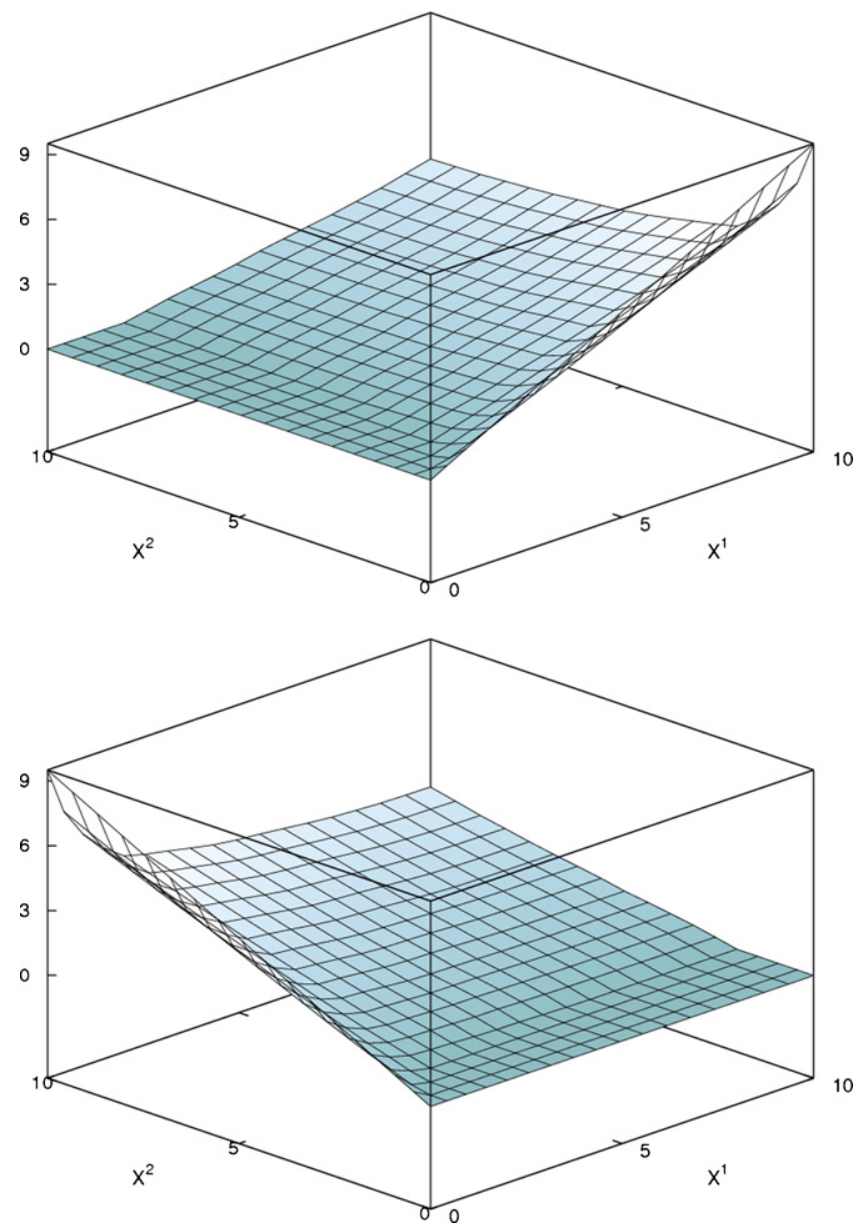

Fig. 3. Investment in commodities 1 (top) and 2 (bottom).

\subsection{The decentralized equilibrium}

We are now ready to connect the equilibrium for the decentralized commodity market defined in Section 2 with the planner's problem. To do so, notice that if one chooses $i: \mathbb{R}_{++}^{M} \rightarrow \mathbb{R}_{+}^{M}$ with $i(x) \in[0, x)$ for all $x$, then for competitive equilibrium to obtain the consumption and price functions $c$ and $p$ are completely determined on $\mathbb{R}_{++}^{M}$ by $i$ via the two equations in (3). As a result, to determine competitive equilibria we can focus on the investment function alone. This leads to the following definition.

Definition 3.1. A function $i: \mathbb{R}_{++}^{M} \rightarrow \mathbb{R}_{+}^{M}$ with $i(x) \in[0, x)$ for all $x$ in $\mathbb{R}_{++}^{M}$ is said to be a competitive equilibrium investment function for the commodity market if $i$ and the induced pricing function $p(x):=\nabla F(x-i(x))$ satisfy (4).

Theorem 3.2. The optimal investment policy I defined in Theorem 3.1 is a competitive equilibrium investment function for the commodity market. Moreover, it is unique in the sense that if $i$ is a competitive equilibrium investment function then $i=I$ on $\mathbb{R}_{++}^{M}$.

Proof. We begin with a definition: let $f_{x}$ be the concave function

$$
f_{x}(\xi):=F(x-\xi)+\rho \int v(\Lambda \xi+z) \phi(\mathrm{d} z), \quad \xi \in[0, x]
$$

It is not difficult to show that $\xi^{*}$ maximizes $f_{x}$ over $[0, x]$ iff

$$
D_{h} f_{x}\left(\xi^{*}\right) \leq 0 \text { for all } h \text { with } \xi^{*}+h \in[0, x]
$$

Now we establish that $I$ defined in Theorem 3.1 is a competitive equilibrium investment function in the sense that $I$ and the induced pricing function $P(x):=\nabla F(x-I(x))$ satisfy

$$
\rho \int\langle P(\Lambda I(x)+z), \Lambda h\rangle \phi(\mathrm{d} z) \leq\langle P(x), h\rangle \quad \forall h \in F_{v}^{I}(x)
$$


for all $x \in \mathbb{R}_{++}^{M}$. To see this, fix $x \in \mathbb{R}_{++}^{M}$ and $h \in F_{v}^{I}(x)$, so that $I(x)+h \in[0, x]$. Since $v$ is differentiable and $x-I(x)$ is interior the directional derivative of $f_{x}$ at $I(x)$ is

$$
D_{h} f_{x}(I(x))=-\langle\nabla F(x-I(x)), h\rangle+\rho \int\langle\nabla v(\Lambda I(x)+z), \Lambda h\rangle \phi(\mathrm{d} z)
$$

Since $I(x)$ maximizes $f_{x}$ over $[0, x]$ and $I(x)+h \in[0, x]$ the inequality in (12) gives

$$
\rho \int\langle\nabla v(\Lambda I(x)+z), \Lambda h\rangle \phi(\mathrm{d} z) \leq\langle\nabla F(x-I(x)), h\rangle
$$

Using $P(x)=\nabla v(x)=\nabla F(x-I(x))$ we obtain (13). Thus $I$ is a competitive equilibrium investment function.

Next we consider uniqueness. Since $f_{x}$ is strictly concave its maximizer $I(x)$ is unique for all $x$. Since the first order condition (12) is necessary and sufficient, it follows that $I$ it the unique function that satisfies (12) for all $x$. Writing this directional derivative in terms of $I$ we see that $I$ is the only function which satisfies

$$
\rho \int\langle\nabla F[\Lambda I(x)+z-I(\Lambda I(x)+z)], \Lambda h\rangle \phi(\mathrm{d} z) \leq\langle\nabla F(x-I(x)), h\rangle \quad \forall h \in F_{v}^{I}(x)
$$

Now suppose that $i$ is a competitive equilibrium investment function, so in particular $p(x)=\nabla F(x-i(x))$ satisfies $(4)$. Then

$$
\rho \int\langle\nabla F[\Lambda i(x)+z-i(\Lambda i(x)+z)], \Lambda h\rangle \phi(\mathrm{d} z) \leq\langle\nabla F(x-i(x)), h\rangle \quad \forall h \in F_{v}^{i}(x)
$$

for all $x \in \mathbb{R}_{++}^{M}$. But we have just established that $I$ is the only function which has this property. Hence $i=I$.

\section{Dynamics}

Next we turn to equilibrium dynamics of $\left(X_{t}\right)_{t \geq 0}$ given the optimal investment policy $I$ defined in (10). The process is Markovian and obeys the stochastic recursive sequence

$$
X_{t+1}=\Lambda I\left(X_{t}\right)+W_{t+1}, \quad\left(W_{t}\right)_{t \geq 0} \stackrel{\text { IID }}{\sim} \phi
$$

The sequence $\left(X_{t}\right)_{t>0}$ can also be seen as the equilibrium time path given in (2), as discussed in Section 2 .

Throughout this section we maintain Assumptions 2.1 and 2.3. Assumption 2.2 is not required. Instead, additional restrictions on the nature of the shock distribution are necessary:

Assumption 4.1. The distribution $\phi$ of the shock $W$ can be represented by a density, which we again denote by $\phi$. The density $\phi$ is continuous everywhere on $\mathbb{R}_{+}^{M}$ and positive on its interior.

Many standard distributions satisfy all of our assumptions, a useful example being the multivariate lognormal density. Heavy tailed densities are also possible, provided that $\mu$ in (1) remains finite. It would appear that the latter assumption is difficult to weaken substantially while maintaining our stability results. ${ }^{10}$

The dynamics in (14) can be encapsulated in the Markov density kernel

$$
q(x, y):=\phi(y-\Lambda I(x)), \quad x, y \in \mathbb{R}_{+}^{M}
$$

Intuitively, $q(x, y)$ is the conditional density of $X_{t+1}$ when $X_{t}=x .{ }^{11}$ If $y \nsucceq \Lambda I(x)$ then $y-\Lambda I(x) \notin \mathbb{R}_{+}^{M}$ and $\phi(y-\Lambda I(x))$ is not defined. For such values of $x$ and $y$ we take $q(x, y)=0$. Alternatively, one can regard $\phi$ as defined on all of $\mathbb{R}^{M}$ and equal to zero on $\mathbb{R}^{M} \backslash \mathbb{R}_{+}^{M}$.

Using standard arguments ${ }^{12}$ we can deduce that if $X_{t}$ has any distribution $\psi_{t}$ (not necessarily a density), then $X_{t+1}$ has a distribution represented by density $\psi_{t+1}$, where

$$
\psi_{t+1}(y)=\int q(x, y) \psi_{t}(\mathrm{~d} x), \quad y \in \mathbb{R}_{+}^{M}
$$

Let $\mathbf{M}$ be a map from the set of distributions on $\mathbb{R}_{+}^{M}$ into the set of densities on $\mathbb{R}_{+}^{M}$ defined by $\psi \mapsto \psi \mathbf{M}$,

$$
\psi \mathbf{M}(y)=\int q(x, y) \psi(\mathrm{d} x), \quad y \in \mathbb{R}_{+}^{M}
$$

\footnotetext{
10 The assumption that $\phi$ is a density can perhaps be relaxed without losing the stability results given below. However, the density assumption is suitable for empirical applications and allows slightly more direct proofs, as well as a more explicit construction of the Markov process generated by the optimal policy.

11 To see this, observe that for any $B \in \mathcal{B}\left(\mathbb{R}_{+}^{M}\right)$ the change of variable $z=y-\Lambda I(x)$ yields $\int \nVdash_{B}(y) \phi(y-\Lambda I(x)) \mathrm{d} y=\int \nVdash_{B}(\Lambda I(x)+z) \phi(z) \mathrm{d} z=\mathbb{P}\{\Lambda I(x)+$ $W \in B\}=\mathbb{P}\left\{X_{t+1} \in B \mid X_{t}=x\right\}$, where $\nVdash_{B}$ denotes the indicator function of $B$.

12 See, for example, Lasota and Mackey (1994) or Stachurski (2002).
} 
This map is called the Markov operator corresponding to $q .{ }^{13}$ In light of (16), the marginal distributions $\left(\psi_{t}\right)$ of $\left(X_{t}\right)$ satisfy $\psi_{t+1}=\psi_{t} \mathbf{M}$. Iterating backwards we obtain $\psi_{t}=\psi_{0} \mathbf{M}^{t}$, where $\mathbf{M}^{t}$ is the $t$ th composition of $\mathbf{M}$ with itself, and, as above, $\psi_{0}$ is the distribution of $X_{0}$.

A distribution $\psi^{*}$ on $\mathbb{R}_{+}^{M}$ is called stationary for the optimal process (14) if $\psi^{*}$ is a fixed point of $\mathbf{M}$. The interpretation is that if $X_{t} \sim \psi^{*}$, then $X_{t+1} \sim \psi^{*} \mathbf{M}=\psi^{*}$, and hence probabilities are unchanged. Since $\mathbf{M}$ maps distributions into densities, any fixed point $\psi^{*}$ of $\mathbf{M}$ must be a density (because $\psi^{*}$ is the image of itself under $\mathbf{M}$ ). Hence in what follows we need concern ourselves only with stationary densities, rather than stationary distributions. For such a stationary density, the defining condition $\psi^{*} \mathbf{M}=\psi^{*}$ translates to

$$
\int q(x, y) \psi^{*}(x) \mathrm{d} x=\psi^{*}(y), \quad y \in S
$$

We measure the distance between densities $\psi$ and $\psi^{\prime}$ according to their deviation with respect to the norm on $L_{1}\left(\mathbb{R}_{+}^{M}\right)$ :

$$
\mathrm{d}_{1}\left(\psi, \psi^{\prime}\right):=\int\left|\psi(x)-\psi^{\prime}(x)\right| \mathrm{d} x
$$

By Scheffè's Identity, we also have

$$
d_{1}\left(\psi, \psi^{\prime}\right)=\sup _{|h| \leq 1}\left|\int h(x) \psi(x) \mathrm{d} x-\int h(x) \psi^{\prime}(x) \mathrm{d} x\right|
$$

Here the supremum is with respect to all Borel measurable bounded functions with supremum norm less than $1 .{ }^{14}$

To state our results, we introduce two classes $\mathcal{H}_{s}$ and $\mathcal{G}_{s}$

$$
\begin{aligned}
& \mathcal{H}_{s}:=\left\{h \in m \mathcal{B}\left(\mathbb{R}_{+}^{M}\right):|h(x)| \leq\|x\|+s \quad \forall x \in \mathbb{R}_{+}^{M}\right\} \quad(s \geq 1) \\
& \mathcal{G}_{s}:=\left\{h \in m \mathcal{B}\left(\mathbb{R}_{+}^{M}\right): h(x)^{2} \leq\|x\|+s \quad \forall x \in \mathbb{R}_{+}^{M}\right\} \quad(s \geq 1)
\end{aligned}
$$

where $m \mathcal{B}\left(\mathbb{R}_{+}^{M}\right)$ is the Borel measurable, real-valued functions on $\mathbb{R}_{+}^{M}$. We can now state the main stability result of the paper.

Theorem 4.1. The following statements are true:

(1) The optimal process (14) has a unique stationary density $\psi^{*}$.

(2) The stationary density $\psi^{*}$ satisfies $\int\|x\| \psi^{*}(\mathrm{~d} x)<\infty$.

(3) If $\psi_{0}$ is any distribution with $\int\|x\| \psi_{0}(\mathrm{~d} x)<\infty$, then for all $s \geq 1$ there is an $M<\infty$ and a $\beta<1$ such that, $\forall t \in \mathbb{N}$,

$$
\sup _{h \in \mathcal{H}_{s}}\left|\int h(x) \psi_{0} \mathbf{M}^{t}(x) \mathrm{d} x-\int h(x) \psi^{*}(x) \mathrm{d} x\right| \leq \beta^{t} M
$$

We present several corollaries to the theorem:

Corollary 4.1. Let $\left(X_{t}\right)_{t \geq 0}$ be the optimal process starting at $x_{0} \in \mathbb{R}_{+}^{M}$. For any such $x_{0}$, the density $\psi_{t}$ of $X_{t}$ converges in $L_{1}\left(\mathbb{R}_{+}^{M}\right)$ to $\psi^{*}$ at a geometric rate.

For the next corollary some additional notation is useful. Let $\left(X_{t}^{*}\right)_{t \geq 0}$ be a stationary version of the process. That is, $X_{t+1}^{*}=\Lambda I\left(X_{t}^{*}\right)+W_{t+1}$ and $X_{0}^{*} \sim \psi^{*}$. Given $h: \mathbb{R}_{+}^{M} \rightarrow \mathbb{R}$, we set

$$
\begin{aligned}
& m_{h}^{*}:=\int h(x) \psi^{*}(x) \mathrm{d} x=\mathbb{E} h\left(X_{0}^{*}\right) \\
& v_{h}^{*}:=\mathbb{E}\left[h\left(X_{0}^{*}\right)-m_{h}^{*}\right]^{2}+2 \sum_{t \geq 1} \mathbb{E}\left[h\left(X_{0}^{*}\right)-m_{h}^{*}\right]\left[h\left(X_{t}^{*}\right)-m_{h}^{*}\right]
\end{aligned}
$$

Corollary 4.2. Let $\psi_{0}$ be an arbitrary in initial condition and let $\left(X_{t}\right)_{t \geq 0}$ be the process starting at $X_{0} \sim \psi_{0}$. If $h \in \mathcal{H}_{s}$ for some $s \geq 1$, then $m_{h}^{*}$ is finite, and

$$
\frac{1}{n} \sum_{t=1}^{n} h\left(X_{t}\right) \rightarrow m_{h}^{*}, \quad \mathbb{P}-\text { a.s. as } n \rightarrow \infty
$$

\footnotetext{
13 As is traditional, $\mathbf{M}$ acts on distributions to the left.

${ }^{14}$ From (19) it is easy to see that $L_{1}$ convergence of densities implies uniform (and hence weak) convergence of distribution functions.
} 


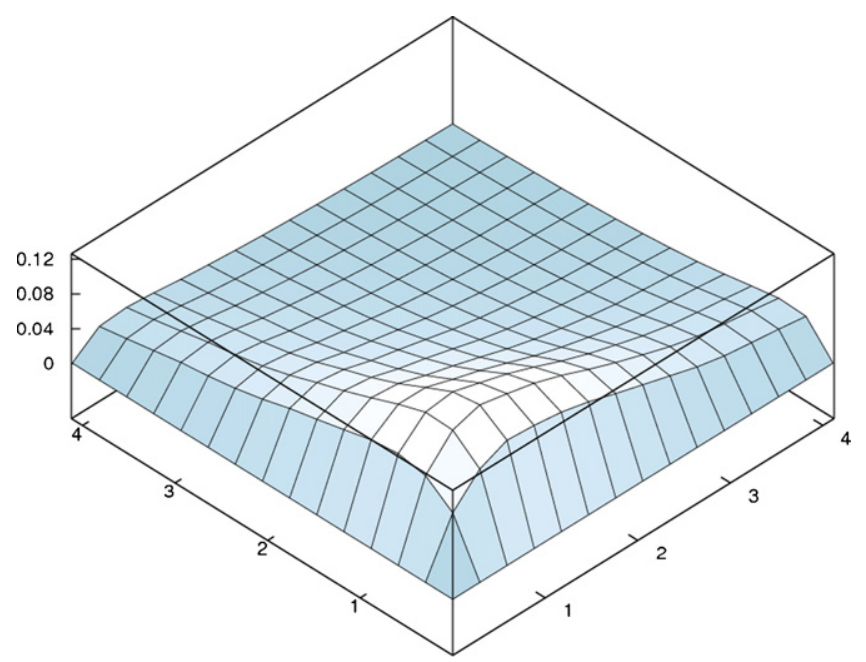

Fig. 4. Approximation $\psi_{n}^{*}$ of $\psi^{*}$.

If, in addition, $h \in \mathcal{G}_{s}$ for some $s \geq 1$, then $v_{h}^{*}$ is finite, and

$$
\frac{1}{n} \sum_{t=1}^{n} h\left(X_{t}\right) \rightarrow N\left(m_{h}^{*}, v_{h}^{*}\right) \quad \text { in distribution as } n \rightarrow \infty
$$

The two most important consequences of Corollary 4.2 are as follows. First, for any $B \in \mathcal{B}\left(\mathbb{R}_{+}^{M}\right)$ we have $(1 / n) \sum_{t=1}^{n} \mathbf{1}_{B}\left(X_{t}\right) \rightarrow$ $\psi^{*}(B)$, and hence the steady state probability $\psi^{*}(B)$ is approximately equal to the fraction of time that the equilibrium quantity spends in $B$ as the time horizon tends to infinity. This is the standard concept of ergodicity. Second, expectations and probabilities vis-á-vis the stationary distribution can be computed by simulation, appealing to (LLN). For such calculations, (CLT) provides (asymptotic) error bounds.

As an application of the second point, we compute an estimate $\psi_{n}^{*}$ of the stationary density $\psi^{*}$ via conditional Monte Carlo (Glynn and Henderson, 2001). Fix $y \in \mathbb{R}_{+}^{M}$. Since $x \mapsto q(x, y)$ is bounded it is an element of $\mathcal{H}_{1}$. By Corollary 4.2, then, we have

$$
\psi_{n}^{*}(y):=\frac{1}{n} \sum_{t=1}^{n} q\left(X_{t}, y\right) \rightarrow \int q(x, y) \psi^{*}(x) \mathrm{d} x, \quad \mathbb{P}-\text { a.s. as } n \rightarrow \infty
$$

By (18), the right-hand side is precisely $\psi^{*}(y)$, so $\psi_{n}^{*}(y) \rightarrow \psi^{*}(y)$ almost surely for all $y$. Fig. 4 displays an instance of $\psi_{n}^{*}$ for the same parameters as in Fig. 2, where $n=2000$.

\section{Conclusion}

In this paper we introduced a multisector commodity pricing model and described the competitive equilibrium. We indicated how one can solve for equilibrium prices and quantities via a corresponding dynamic program. We also showed that the equilibrium state process is stationary and geometrically ergodic. This sets out a cohesive framework for future empirical analysis.

A number of extensions to our model can be considered. One is to include stochastic demand on the part of firms driven by shocks to production and output prices. Another is correlated shocks for the harvest processes. In terms of the dynamics, the impact of correlated shocks is unclear. While establishing the existence of a stationary distribution should be possible via continuity arguments, stability is highly problematic given the lack of monotonicity and the obvious problems in establishing irreducibility. Such topics are left for future research.

\section{Acknowledgements}

The authors thank Kevin Reffett for many helpful comments. The second author is grateful for financial support from the Japan Society for the Promotion of Science Young Researcher Award. 


\section{Appendix A}

This section collects all remaining proofs. Throughout the proofs we adopt the new notation $\bar{\alpha}:=\max _{1 \leq m \leq M} \alpha^{m}$. Evidently $\|\Lambda x\| \leq \bar{\alpha}\|x\|, \forall x \in \mathbb{R}^{M}$.

\section{A.1. Optimality}

Our first task is to prove Lemma 3.1. Recall our definition of the auxiliary process $\left(Y_{t}\right)_{t \geq 0}$ by $Y_{t+1}=\Lambda Y_{t}+W_{t+1}$ with $Y_{0}=x$. Alternatively, $Y_{t}=\Lambda^{t} x+\sum_{j=1}^{t} \Lambda^{t-j} W_{j}$. Since $F$ is concave there exist positive constants $b_{0}$ and $b_{1}$ such that $F(x) \leq b_{0}+b_{1}\|x\|$ for all $x \in \mathbb{R}_{+}^{M}$. Moreover, $\left\|\Lambda^{k} z\right\| \leq \bar{\alpha}^{k}\|z\|$ for any $z \in \mathbb{R}^{M}$. Consequently,

$$
\mathbb{E} F\left(\Lambda^{t} x+\sum_{j=1}^{t} \Lambda^{t-j} W_{j}\right) \leq b_{0}+b_{1} \mathbb{E}\left\|\Lambda^{t} x+\sum_{j=1}^{t} \Lambda^{t-j} W_{j}\right\| \leq b_{0}+b_{1}\|x\|+\frac{b_{1} \mu}{1-\bar{\alpha}}=: M(x)
$$

Given this bound the finiteness of $\kappa(x)=1+\sum_{t=0}^{\infty} \delta^{t} \mathbb{E} F\left(Y_{t}\right)$ is immediate:

$$
\kappa(x)=1+\sum_{t=0}^{\infty} \delta^{t} \mathbb{E} F\left(\Lambda^{t} x+\sum_{j=1}^{t} \Lambda^{t-j} W_{j}\right) \leq 1+\frac{M(x)}{1-\delta}
$$

That $\kappa$ is increasing follows from monotonicity of $F$. Continuity of $\kappa$ follows from continuity of $F$ and the Dominated Convergence Theorem.

The remainder of the proof of Lemma 3.1 is straightforward. Since $F$ is increasing and $X_{t}-i\left(X_{t}\right) \leq X_{t} \leq Y_{t}$ pointwise on $\Omega$ for any $i \in \mathcal{I}$ we have $v_{i} \leq \kappa$. Since $v(x)$ is defined as $\sup _{i \in \mathcal{I}} v_{i}(x)$ and since $v_{i}(x) \leq \kappa(x)$ for every $i \in \mathcal{I}$ the function $v$ exists and is dominated by $\kappa$. This completes the proof of Lemma 3.1.

Next we turn to the proof of Proposition 3.1. For this proof some extra notation is useful. In particular, for any appropriately integrable function $h$ on $\mathbb{R}_{+}^{M}$ we define the new function $\mathbf{N} h$ by

$$
\mathbf{N} h(x):=\int h(\Lambda x+z) \phi(\mathrm{d} z)
$$

so that $\mathbf{N} h(x)$ is the expectation of $h\left(Y_{t}\right)$ given $Y_{t-1}=x \cdot{ }^{15}$ Evidently $h \leq h^{\prime}$ implies $\mathbf{N} h \leq \mathbf{N} h^{\prime}$, and $\mathbf{N 1}=\mathbf{1}$. We let $\mathbf{N}^{t}$ be the $t$-th iterate, in which case $\mathbf{N}^{t} h(x)$ is the expectation of $h\left(Y_{t}\right)$ given $Y_{0}=x$. In particular, $\mathbf{N}^{t} F(x)=\mathbb{E} F\left(Y_{t}\right)$ for all $t$, and we can express $\kappa$ as

$$
\kappa(x)=1+\sum_{t=0}^{\infty} \delta^{t} \mathbb{E} F\left(Y_{t}\right)=1+\sum_{t=0}^{\infty} \delta^{t} \mathbf{N}^{t} F(x)
$$

To prove that $T$ is well-defined and contracting on $b_{\kappa} \mathcal{B} \mathbb{R}_{+}^{M}$ we need

Lemma Appendix A.1. For any $x \in \mathbb{R}_{+}^{M}$ the weight function $\kappa$ satisfies

$$
\sup _{0 \leq \xi \leq x} \int \kappa(\Lambda \xi+z) \phi(\mathrm{d} z) \leq \frac{\kappa(x)}{\delta}
$$

Proof. Pick any $x \in \mathbb{R}_{+}^{M}$. Since $\kappa$ is increasing,

$$
\sup _{0 \leq \xi \leq x} \int \kappa(\Lambda \xi+z) \phi(\mathrm{d} z) \leq \int \kappa(\Lambda x+z) \phi(\mathrm{d} z)=\mathbf{N} \kappa(x)
$$

\footnotetext{
15 The operator $\mathbf{N}$ corresponds to $T$ in Stokey, Lucas and Prescott (1989, $§$ 8.1).
} 
But from the definitions and the Dominated Convergence Theorem,

$$
\begin{aligned}
\mathbf{N} \kappa(x) & =1+\mathbf{N} \sum_{t=0}^{\infty} \delta^{t} \mathbf{N}^{t} F(x) \\
& =1+\sum_{t=0}^{\infty} \delta^{t} \mathbf{N}^{t+1} F(x) \\
& =1+(1 / \delta) \sum_{t=0}^{\infty} \delta^{t+1} \mathbf{N}^{t+1} F(x) \\
& \leq 1+(1 / \delta) \sum_{t=0}^{\infty} \delta^{t} \mathbf{N}^{t} F(x) \leq(1 / \delta)+(1 / \delta) \sum_{t=0}^{\infty} \delta^{t} \mathbf{N}^{t} F(x)
\end{aligned}
$$

This last expression is just $(1 / \delta) \kappa(x)$, and the proof is done.

Using Lemma A.1 we can show that the Bellman operator $T$ does send $b_{\kappa} \mathcal{B R}_{+}^{M}$ into itself-in particular, $T w$ is $\kappa$-bounded whenever $w$ is. Indeed, for any $w \in b_{\kappa} \mathcal{B} \mathbb{R}_{+}^{M}$ we have

$$
\begin{aligned}
|T w(x)| & \leq \sup _{0 \leq \xi \leq x}\left|F(x-\xi)+\rho \int w(\Lambda \xi+z) \phi(\mathrm{d} z)\right| \\
& \leq F(x)+\rho \sup _{0 \leq \xi \leq x} \int|w(\Lambda \xi+z)| \phi(\mathrm{d} z) \\
& \leq F(x)+\rho\|w\|_{\kappa} \sup _{0 \leq \xi \leq x} \int \kappa(\Lambda \xi+z) \phi(\mathrm{d} z) \\
& \leq F(x)+\frac{\rho\|w\|_{\kappa} \kappa(x)}{\delta}
\end{aligned}
$$

Since $F(x) \leq \kappa(x)$ it follows that for any $x \in \mathbb{R}_{+}^{M}$ we have

$$
\frac{|T w(x)|}{\kappa(x)} \leq 1+\frac{\rho\|w\|_{\kappa}}{\delta}
$$

Thus $T w$ is $\kappa$-bounded, as was to be shown.

In order to prove that $T$ is a contraction of modulus $\gamma=\rho / \delta$ we use the following extension of Blackwell's sufficient condition, which is proved in Hernández-Lerma and Lasserre (1999, Proposition 7.2.9).

Lemma Appendix A.2. If $T$ is monotone and, for any $c \in \mathbb{R}_{+}$and $w \in b_{\kappa} \mathcal{B} \mathbb{R}_{+}^{M}$,

$$
T(w+c \kappa) \leq T w+\gamma c \kappa
$$

then $T$ is $a\|\cdot\|_{\kappa}$-contraction of modulus $\gamma$ on $b_{\kappa} \mathcal{B} \mathbb{R}_{+}^{M}$.

By monotonicity is meant that for any pair $w, w^{\prime} \in b_{\kappa} \mathcal{B} \mathbb{R}_{+}^{M}$ with $w \leq w^{\prime}$ we have $T w \leq T w^{\prime}$. This property is easily shown and the proof is omitted. To verify (20), observe that

$$
T(w+c \kappa)(x)=\sup _{0 \leq \xi \leq x}\left\{F(x-\xi)+\rho \int w(\Lambda \xi+z) \phi(\mathrm{d} z)+c \rho \int \kappa(\Lambda \xi+z) \phi(\mathrm{d} z)\right\} \leq T w(x)+c \rho \sup _{0 \leq \xi \leq x} \int \kappa(\Lambda \xi+z) \phi(\mathrm{d} z)
$$

In light of Lemma A.1 we have

$$
\begin{aligned}
& \sup _{0 \leq \xi \leq x} \int \kappa(\Lambda \xi+z) \phi(\mathrm{d} z)=\int \kappa(\Lambda x+z) \phi(\mathrm{d} z) \leq \frac{\kappa(x)}{\delta} \\
& \therefore \quad T(w+c \kappa)(x) \leq T w(x)+\frac{\rho}{\delta} c \kappa(x)
\end{aligned}
$$

Since $\gamma=\rho / \delta$ the proof is complete.

The only claim in Proposition 3.1 that remains to be verified is that $T$ maps the set of continuous $\kappa$-bounded functions $b_{\kappa} c \mathbb{R}_{+}^{M}$ into itself. In particular, we need to check that if $w$ is continuous $\kappa$-bounded then $T w$ is continuous. To see this, pick any such $w$. In view of Berge's Theorem of the Maximum, the function

$$
T w(x)=\sup _{0 \leq \xi \leq x}\left\{F(x-\xi)+\rho \int w(\Lambda \xi+z) \phi(\mathrm{d} z)\right\}
$$


will be continuous provided that

$$
(x, \xi) \mapsto F(x-\xi)+\rho \int w(\Lambda \xi+z) \phi(\mathrm{d} z)
$$

is jointly continuous on $\left\{(x, \xi): x \in \mathbb{R}_{+}^{M}, 0 \leq \xi \leq x\right\}$. The only nontrivial assertion is that if $\left(\xi_{n}\right) \subset \mathbb{R}_{+}^{M}$ with $\xi_{n} \rightarrow \xi$, then

$$
\int w\left(\Lambda \xi_{n}+z\right) \phi(\mathrm{d} z) \rightarrow \int w(\Lambda \xi+z) \phi(\mathrm{d} z)
$$

In view of continuity of $w$ and the Dominated Convergence Theorem, it is sufficient to show that $\left|w\left(\Lambda \xi_{n}+z\right)\right|$ is dominated pointwise by some integrable function for all $n$. But if $x$ is any vector with $\xi_{n} \leq x$ for all $n$, then for any $n \in \mathbb{N}$ and any $z \in \mathbb{R}_{+}^{M}$ we have

$$
\left|w\left(\Lambda \xi_{n}+z\right)\right| \leq\|w\|_{\kappa} \kappa\left(\Lambda \xi_{n}+z\right) \leq \kappa(\Lambda x+z)
$$

The integral of the right-hand side is finite by Lemma A.1. This completes the proof of Proposition 3.1.

Next we prove Theorem 3.1. Since $T$ is a $\|\cdot\|_{\kappa}$-contraction on the Banach space $b_{\kappa} \mathcal{B R}_{+}^{M}$ it follows that $T$ has a unique fixed point $\bar{w} \in b_{\kappa} \mathcal{B} \mathbb{R}_{+}^{M}$ and $\left\|T^{n} w-\bar{w}\right\|_{\kappa} \rightarrow 0$ as $n \rightarrow \infty$ for any $w \in b_{\kappa} \mathcal{B} \mathbb{R}_{+}^{M}$. Moreover, $\bar{w} \in b_{\kappa} c \mathbb{R}_{+}^{M}$ and is therefore continuous, as $b_{\kappa} c \mathbb{R}_{+}^{M}$ is a closed subset of $b_{\kappa} \mathcal{B} \mathbb{R}_{+}^{M}$ on which $T$ is invariant. The proof that $\bar{w}$ is in fact equal to the value function $v$ is almost identical to the standard argument (i.e., the argument for bounded rewards) and is omitted.

Existence of a maximizer $I(x)$ for each $x$ follows from continuity of the objective and compactness of the constraint. Continuity of $I$ follows from Berge's Theorem of the Maximum. That $v$ is strictly increasing and strictly concave can be proved by a small modification of the usual technique. ${ }^{16}$

Let us now consider the proof of Proposition 3.2. First we show that if $x \gg 0$ then $c(x):=x-I(x) \gg 0$. To this end, pick any $x \gg 0$ and let $c:=c(x)$. Suppose instead that $c \in \partial \mathbb{R}_{+}^{M}$. Let $d:=x-c$. By Assumption 2.2 we have

$$
\lim _{\theta \downarrow 0} \frac{F(c+\theta d)-F(c)}{\theta}=\infty
$$

For $v$ the value function, define the new function $g$ by

$$
g(s)=\rho \int v(\Lambda(x-s)+z) \phi(\mathrm{d} z)
$$

There is no difficulty in checking that $g$ is well-defined and concave on $(-\infty, x]$. It follows that

$$
h(\theta):=\frac{g(c+\theta d)-g(c)}{\theta}
$$

is well-defined and (by concavity) decreasing on $(-\infty, 0) \cup(0,1]$. As a result, the $\operatorname{limit}_{\lim } \lim _{\downarrow} h(\theta)$ exists and is finite. ${ }^{17}$ Now since $c$ is optimal, and since the alternative $c+\theta d$ is less than $x$ and therefore feasible at $x$ for $\theta \in(0,1]$, we must have

$$
F(c)+\rho \int v(\Lambda(x-c)+z) \phi(\mathrm{d} z) \geq F(c+\theta d)+\rho \int v(\Lambda(x-(c+\theta d))+z) \phi(\mathrm{d} z)
$$

Using the function $g$ this can be rewritten as

$$
F(c)+g(c) \geq F(c+\theta d)+g(c+\theta d)
$$

Rearranging and dividing through by $\theta$ gives

$$
\frac{F(c+\theta d)-F(c)}{\theta} \leq-\frac{g(c+\theta d)-g(c)}{\theta}=-h(\theta)
$$

The left-hand side diverges to infinity as $\theta \downarrow 0$, while the right-hand side converges to a finite constant. This contradicts our assumption that $c \in \partial \mathbb{R}_{+}^{M}$, and we conclude that $c=x-I(x) \gg 0$.

To complete the proof of Proposition 3.2 we show that $v$ is differentiable on $\mathbb{R}_{++}^{M}$ and satisfies the envelope condition $\nabla v(x)=\nabla F(x-I(x))$. We use the well-known techniques developed by Mirman and Zilcha (1975) and Benveniste and Scheinkman (1979, Lemma 1). In particular, if $x \in \mathbb{R}_{++}^{M}$ and $w$ is any concave differentiable function defined on a neighborhood $N$ of $x$ and satisfying $w(x)=v(x)$ and $w(y) \leq v(y)$ for all $y \in N$, then $v$ is differentiable at $x$ and $\nabla v(x)=\nabla w(x)$.

\footnotetext{
${ }^{16}$ It is easy to show that $T$ maps the set $\mathcal{C}$ of increasing concave functions in $b_{\kappa} C \mathbb{R}_{+}^{M}$ into itself. Moreover, a simple argument shows that $\|\cdot\|_{\kappa}$-convergence implies pointwise convergence, which in turn preserves monotonicity and concavity. Hence $\mathcal{C}$ is $\|\cdot\|_{\kappa}$-closed. As $T: \mathcal{C} \rightarrow \mathcal{C}$ and $\mathcal{C}$ is $\|\cdot\|_{\kappa}$-closed we have $v \in \mathcal{C}$. Finally, $T$ maps elements of $\mathcal{C}$ into strictly increasing, strictly concave functions in $\mathcal{C}$, so $v$ is strictly increasing and strictly concave (because $T v=v$ ).

17 The value $h(\theta)$ increases monotonically as $\theta \downarrow 0$ and is bounded by $h(-1)$.
} 
Although investment is not interior, the interiority of demand is sufficient for this technique to work. To see this, pick any $x_{0} \gg 0$, and let $i_{0}:=I\left(x_{0}\right)$. Since $c\left(x_{0}\right)=x_{0}-i_{0} \gg 0$ we have $x_{0} \gg i_{0}$, and there exists an open neighborhood $N$ of $x_{0}$ with $N \subset \mathbb{R}_{++}^{M}$ and $i_{0} \leq x$ for all $x \in N$. On the set $N$ define

$$
w(x)=F\left(x-i_{0}\right)+\rho \int v\left(\Lambda i_{0}+z\right) \phi(\mathrm{d} z)
$$

Note that $w$ is well-defined on $N$, as $i_{0} \leq x$ for all $x \in N$. In addition, for each $x \in N$ investment $i_{0}$ is feasible, so

$$
w(x) \leq v(x)=F(x-I(x))+\rho \int v(\Lambda I(x)+z) \phi(\mathrm{d} z)
$$

Evidently $w$ is concave and $v\left(x_{0}\right)=w\left(x_{0}\right)$. Finally, $w$ is differentiable at $x_{0}$ with $\nabla w\left(x_{0}\right)=\nabla F\left(x_{0}-I\left(x_{0}\right)\right)$. Hence $\nabla v\left(x_{0}\right)=$ $\nabla F\left(x_{0}-I\left(x_{0}\right)\right)$.

\section{A.2. Dynamics}

Now we turn to dynamics with a view to proving Theorem 4.1. Recall the definition of $q$ in (15). With respect to this $q$ we define $q$-small sets, aperiodicity and irreducibility. ${ }^{18}$

Definition Appendix A.1. A set $C \in \mathcal{B}\left(\mathbb{R}_{+}^{M}\right)$ is called $q$-small if

$$
\exists g \in L_{1}\left(\mathbb{R}_{+}^{M}\right) \text { such that } g \geq 0, g \neq 0 \text { and } \forall x \in C, \quad q(x, \cdot) \geq g
$$

If such set $C$ exists and $\int_{C} g>0$, then $\left(X_{t}\right)_{t \geq 0}$ is called aperiodic. ${ }^{19}$

Definition Appendix A.2. The optimal process $\left(X_{t}\right)_{t \geq 0}$ is called irreducible if, $\forall x_{0} \in \mathbb{R}_{+}^{M}$ and $\forall B \in \mathcal{B}\left(\mathbb{R}_{+}^{M}\right)$ with $\lambda(B)>0$, the process $\left(X_{t}\right)_{t \geq 0}$ started at $X_{0} \equiv x_{0}$ satisfies $\mathbb{P} \cup_{t \geq 1}\left\{X_{t} \in B\right\}>0$.

Let $s \geq 1$, and let $V(x):=\|x\|+s$. By Meyn and Tweedie (1993), Theorem 16.1.2, if $\left(X_{t}\right)_{t \geq 0}$ is aperiodic, irreducible and possesses a $q$-small set $C$ such that

$$
\int V(y) q(x, y) \mathrm{d} y \leq \gamma V(x)+b \mathbf{1}_{C}(x), \quad x \in \mathbb{R}_{+}^{M}
$$

for some $\gamma<1$ and $b<\infty$, then $\left(X_{t}\right)_{t \geq 0}$ is $V$-uniformly ergodic. In particular, there exists a unique stationary distribution (in this case a density) $\psi^{*}$; the density $\psi^{*}$ satisfies $\int V(x) \psi^{*}(x) \mathrm{d} x<\infty$; and, moreover, there is a $\beta<1$ and $N<\infty$ such that

$$
\sup _{|h| \leq V}\left|\int h(y) \delta_{x} \mathbf{M}^{t}(y) \mathrm{d} y-\int h(y) \psi^{*}(y) \mathrm{d} y\right| \leq \beta^{t} N V(x)
$$

for all $t \in \mathbb{N}$ and all $x \in \mathbb{R}_{+}^{M}$. Here $\delta_{x}$ is the distribution concentrated at $x$, so that $\delta_{x} \mathbf{M}^{t}$ is the density of $X_{t}$ when $X_{0} \equiv x$. Below we establish that $\left(X_{t}\right)_{t \geq 0}$ is $V$-uniformly ergodic for any choice of the constant $s \in[1, \infty)$. The conclusions of Theorem 4.1 then follow in a straightforward way. Parts (1) and (2) are immediate. To see that (3) is true, pick any $s \geq 1$ and any distribution $\psi_{0}$ such that $\int\|x\| \psi_{0}(\mathrm{~d} x)$ is finite. Now take any $h \in \mathcal{H}_{s}$. Consider the term

$$
\left|\int h(y) \psi_{0} \mathbf{M}^{t}(y) \mathrm{d} y-\int h(y) \psi^{*}(y) \mathrm{d} y\right|=\left|\int h(y)\left[\int \delta_{x} \mathbf{M}^{t}(y) \psi_{0}(\mathrm{~d} x)\right] \mathrm{d} y-\int h(y) \psi^{*}(y) \mathrm{d} y\right|
$$

Since $|h| \leq V=\|\cdot\|+s$, Eq. (23) implies that the right-hand side is dominated by

$$
\begin{aligned}
& \int\left|\int h(y) \delta_{x} \mathbf{M}^{t}(y) \mathrm{d} y-\int h(y) \psi^{*}(y) \mathrm{d} y\right| \psi_{0}(\mathrm{~d} x) \leq \beta^{t} N \int V(x) \psi_{0}(\mathrm{~d} x) \\
& \therefore\left|\int h(y) \psi_{0} \mathbf{M}^{t}(y) \mathrm{d} y-\int h(y) \psi^{*}(y) \mathrm{d} y\right| \leq \beta^{t} N\left(\int\|x\| \psi_{0}(\mathrm{~d} x)+s\right)
\end{aligned}
$$

As $h$ is an arbitrary element of $\mathcal{H}_{s}$ the conclusion of Theorem 4.1 follows.

In summary, to prove Theorem 4.1, it is sufficient to establish that for any $s \geq 1$, the optimal process $\left(X_{t}\right)_{t>0}$ is irreducible, aperiodic and possesses a small set $C$ such that (22) holds for $V(x)=\|x\|+s$. We begin with irreducibility:

Proposition Appendix A.1. The optimal process $\left(X_{t}\right)_{t \geq 0}$ is irreducible.

Proof. Fix $x_{0} \in \mathbb{R}_{+}^{M}$ and $B \in \mathcal{B}\left(\mathbb{R}_{+}^{M}\right)$ with $\lambda(B)>0$. Let $\mathbf{1} \in \mathbb{R}_{+}^{M}$ be the vector of ones. Evidently one can select a strictly positive scalar $a$ with the property $\lambda([a 1, \infty) \cap B)>0$. To prove Proposition A.1 we need the following two lemmas concerning $a$.

\footnotetext{
18 See Meyn and Tweedie (1993) for more details on these concepts.

19 Our definitions of small sets and aperiodicity are slightly stronger than the standard definitions. See Meyn and Tweedie (1993, Chapter 5).
} 
Lemma Appendix A.3. If $x \in(0, a 1]$, then

$$
\int_{B} q(x, y) \mathrm{d} y=\int_{B} \phi(y-\Lambda I(x)) \mathrm{d} y>0
$$

Proof. For $y \in[a \mathbf{1}, \infty)$, the interiority of $x$ implies that

$$
\begin{aligned}
& y \geq a \mathbf{1} \geq x \gg \Lambda x \geq \Lambda I(x) \\
& \therefore \quad \phi(y-\Lambda I(x))>0
\end{aligned}
$$

Since $[a \mathbf{1}, \infty) \cap B$ has positive Lebesgue measure, it follows that

$$
\begin{aligned}
& \int_{B \cap[a \mathbf{1}, \infty)} \phi(y-\Lambda I(x)) \mathrm{d} y>0 \\
& \therefore \quad \int_{B} \phi(y-\Lambda I(x)) \mathrm{d} y \geq \int_{B \cap[a 1, \infty)} \phi(y-\Lambda I(x)) \mathrm{d} y>0
\end{aligned}
$$

Hence $X_{t} \in(0, a \mathbf{1}]$ implies $X_{t+1} \in B$ with positive probability.

Lemma Appendix A.4. For each $x_{0} \in \mathbb{R}_{+}^{M}$ there is an $n \geq 0$ such that $\mathbb{P}\left\{X_{n} \in(0, a \mathbf{1}]\right\}>0$, where $\left(X_{t}\right)_{t \geq 0}$ is the process starting at $x_{0}$.

Proof. Let $\|x\|_{\infty}:=\max _{1 \leq m \leq M} x^{m}$ for any $x=\left(x^{m}\right)_{m=1}^{M} \in \mathbb{R}_{+}^{M}$. Note that $\|\Lambda x\|_{\infty} \leq \bar{\alpha}\|x\|_{\infty}$ holds for any $x$. Note also that $\|\cdot\| \infty$ is consistent with the ordering on $\mathbb{R}_{+}^{M}$, in the sense that $x \leq y$ implies $\|x\|_{\infty} \leq\|y\|_{\infty}$.

Since $a>0$ and $\bar{\alpha}<1$, clearly we can choose an $n \geq 0$ and a $z_{0} \gg 0$ such that

$$
\bar{\alpha}^{n}\left\|x_{0}\right\|_{\infty}+\left\|z_{0}\right\|_{\infty} \frac{1}{1-\bar{\alpha}} \leq a
$$

Let $E$ be the event $\cap_{t \leq n}\left\{W_{t} \leq z_{0}\right\}$. Evidently $E$ has positive probability, so it suffices to prove that $X_{n} \leq a \mathbf{1}$ on $E$. To this end, observe that on $E$ we have $X_{t} \leq \Lambda X_{t-1}+z_{0}$ for $t=1, \ldots, n$.

$$
\begin{array}{ll}
\therefore & X_{n} \leq \Lambda^{n} x_{0}+\Lambda^{0} z_{0}+\Lambda^{1} z_{0}+\cdots+\Lambda^{n-1} z_{0} \\
\therefore & \left\|X_{n}\right\|_{\infty} \leq \bar{\alpha}^{n}\left\|x_{0}\right\|_{\infty}+\left\|z_{0}\right\|_{\infty} \frac{1}{1-\bar{\alpha}} \leq a \\
\therefore & X_{n} \leq a \mathbf{1}
\end{array}
$$

The proof of Lemma A.4 is now complete.

It remains to complete the proof of Proposition A.1. Regarding irreducibility, fix $x_{0} \in \mathbb{R}_{+}^{M}$ and let $\left(X_{t}\right)_{t \geq 0}$ be the process starting at $x_{0}$. By Lemma A.4 there is an $n \geq 0$ such that $\mathbb{P}\left\{X_{n} \in(0, a 1]\right\}>0$. We show that $\mathbb{P}\left\{X_{n+1} \in B\right\}>0$, which implies irreducibility. Note that

$$
\mathbb{P}\left\{X_{n+1} \in B\right\} \geq \mathbb{P}\left[\left\{X_{n} \in(0, a]\right\} \cap\left\{X_{n+1} \in B\right\}\right]=\mathbb{P}\left[\mathbb{P}\left[\left\{X_{n} \in(0, a]\right\} \cap\left\{X_{n+1} \in B\right\} \mid \mathcal{F}_{n}\right]\right]
$$

and

$$
\mathbb{P}\left[\left\{X_{n} \in(0, a]\right\} \mathbb{P}\left[\left\{X_{n+1} \in B\right\} \mid \mathcal{F}_{n}\right]\right]=\mathbb{P}\left[\left\{X_{n} \in(0, a]\right\} \int_{B} q\left(X_{n}, y\right) \mathrm{d} y\right]
$$

where $\mathcal{F}_{t}:=\sigma\left(W_{1}, \ldots, W_{t}\right)$. This last term is strictly positive, because $\mathbb{P}\left\{X_{n} \in[0, a)\right\}>0$ by the definition of $n$, and on $\left\{X_{n} \in(0, a]\right\}$ the integral is strictly positive (Lemma A.3). The proof is done.

Next we address the existence of small sets.

Lemma Appendix A.5. All bounded Borel measurable subsets of $\mathbb{R}_{+}^{M}$ are q-small.

Proof. Since measurable subsets of small sets are small, it suffices to prove that all sets of the form $C=[0, c], c \gg 0$, are $q$-small. Pick any $c \gg 0$ and set $C:=[0, c]$. Let $\alpha^{\prime}$ be any number satisfying $\bar{\alpha}<\alpha^{\prime}<1$, and set

$$
K:=\left\{(x, y) \in \mathbb{R}_{+}^{M} \times \mathbb{R}_{+}^{M}: x \in C, \quad \alpha^{\prime} c \leq y \leq c\right\}
$$

For $(x, y) \in K$ we have

$$
y-\Lambda I(x) \geq y-\Lambda x \geq y-\Lambda c \geq \alpha^{\prime} c-\Lambda c \gg 0
$$


Since $\phi(z)>0$ whenever $z \gg 0$, it follows that $\phi(y-\Lambda I(x))>0$. Combining this observation with the compactness of $K$ and the continuity of $\phi$ it follows that $\epsilon:=\min \{\phi(y-\Lambda I(x)):(x, y) \in K\}$ is strictly positive.

Let $g:=\epsilon \mathbf{1}_{\left[\alpha^{\prime} c, c\right]}$. Since $\epsilon>0, c \gg 0$ and $\alpha^{\prime}<1$, the function $g$ is nontrivial. We claim that $g$ satisfies (21). To see this, pick any $x \in C$. If $y \notin\left[\alpha^{\prime} c, c\right]$ then $g(y)=0$, and (21) must hold. On the other hand, if $y \in\left[\alpha^{\prime} c, c\right]$, then $(x, y) \in K$, and, by the definition of $\epsilon, \phi(y-\Lambda I(x)) \geq \epsilon=g(y)$. Either way we have $q(x, y)=\phi(y-\Lambda I(x)) \geq g(y)$ as claimed.

For this $C$ and $g$ we have $\int_{C} g(y) \mathrm{d} y=\epsilon \lambda\left(\left[\alpha^{\prime} c, c\right]\right)>0$, implying $\left(X_{t}\right)_{t \geq 0}$ is aperiodic. To prove Theorem 4.1, then, it remains only to show that there exists a $q$-small set $C$ such that

$$
\int V(y) q(x, y) \mathrm{d} y \leq \gamma V(x)+b \mathbf{1}_{C}(x), \quad x \in \mathbb{R}_{+}^{M}
$$

for some $\gamma<1$ and $b<\infty$, where $V(x)=\|x\|+s$. Using the change of variable $z=y-\Lambda I(x)$,

$$
\int V(y) q(x, y) \mathrm{d} y=\int V(y) \phi(y-\Lambda I(x)) \mathrm{d} y=\int V(\Lambda I(x)+z) \phi(z) \mathrm{d} z
$$

From $V(x)=\|x\|+s$ this gives

$$
\begin{aligned}
\int V(y) q(x, y) \mathrm{d} y & =\int\|\Lambda I(x)+z\| \phi(z) \mathrm{d} z+s \\
& \leq \bar{\alpha}\|x\|+\int\|z\| \phi(z) \mathrm{d} z+s \\
& \leq \bar{\alpha} V(x)+b, \quad b:=\int\|z\| \phi(z) \mathrm{d} z+s
\end{aligned}
$$

The constant $b$ is finite by Assumption 2.3.

Let $\gamma$ be any number in $(\bar{\alpha}, 1)$. Choose a vector $c \gg 0$ such that

$$
x \notin c \Rightarrow \bar{\alpha}+\frac{b}{V(x)} \leq \gamma
$$

It follows that if $x \not c$, then

$$
\int V(y) q(x, y) \mathrm{d} y \leq \bar{\alpha} V(x)+b \leq \gamma V(x)
$$

Defining $C:=[0, c]$ now gives

$$
\int V(y) q(x, y) \mathrm{d} y \leq \gamma V(x)+b \mathbf{1}_{C}(x), \quad x \in \mathbb{R}_{+}^{M}
$$

as required. As $C$ is $q$-small (Lemma A.5) the proof is done.

Finally, let us turn to the proofs of Corollary 4.1 and Corollary 4.2.

Proof of Corollary 4.1. The proof is almost trivial: let $x_{0} \in \mathbb{R}_{0}^{M}$, and let $\psi_{0}=\delta_{x_{0}}$. Evidently the conditions of Theorem 4.1 part (3) hold, and

$$
\sup _{h \in \mathcal{H}_{1}}\left|\int h(x) \psi_{0} \mathbf{M}^{t}(x) \mathrm{d} x-\int h(x) \psi^{*}(x) \mathrm{d} x\right|=O\left(\beta^{t}\right)
$$

for some $\beta \in(0,1)$. Since $\mathcal{H}_{1}$ contains all Borel measurable real-valued functions $h$ with $|h| \leq 1$, it follows from (19) that $d_{1}\left(\psi_{t}, \psi^{*}\right)=O\left(\beta^{t}\right)$.

Proof of Corollary 4.2. Since $\left(X_{t}\right)_{t \geq 0}$ has been shown to be $V$-uniformly ergodic, both the LLN and the CLT results are immediate from Meyn and Tweedie (1993, Theorem 17.0.1).

\section{References}

Alvarez, F., Stokey, N.L., 1998. Dynamic programming with homogeneous functions. Journal of Economic Theory 82, 167-189.

Benveniste, L.M., Scheinkman, J.A., 1979. On the differentiability of the value function in dynamic models of economics. Econometrica 47, 727-732.

Bobenrieth, E.S.A., Bobenrieth, J.R.A., Wright, B.D., 2002. A commodity price process with a unique continuous invariant distribution having infinite mean.

Econometrica 70, 1213-1219.

Boyd, J.H., 1990. Recursive utility and the Ramsey problem. Journal of Economic Theory 50, 326-345.

Chambers, M.J., Bailey, R.E., 1996. A theory of commodity price fluctuations. Journal of Political Economy 104, 924-957.

Deaton, A., Laroque, G., 1992. On the behavior of commodity prices. Review of Economic Studies 59, 1-23.

Deaton, A., Laroque, G., 1996. Competitive storage and commodity price dynamics. Journal of Political Economy 104, 896-923.

Duffie, D., Singleton, K., 1993. Simulated moments estimation of Markov models of asset prices. Econometrica 61, 929-952.

Glynn, P.W., Henderson, S.G., 2001. Computing densities for Markov chains via simulation. Mathematics of Operations Research $26,375-400$.

Hernández-Lerma, O., Lasserre, J.B., 1999. Further Topics on Discrete Time Markov Control Processes. Springer-Verlag, London. 
Lasota, A., Mackey, M.C., 1994. Chaos, Fractals and Noise: Stochastic Aspects of Dynamics. Springer-Verlag, New York.

Le Van, C., Morhaim, L., 2002. Optimal growth models with bounded or unbounded returns: a unifying approach. Journal of Economic Theory 105, 158-187.

Meyn, S.P., Tweedie, R.L., 1993. Markov Chains and Stochastic Stability. Springer-Verlag, London.

Mirman, L.J., Zilcha, I., 1975. On optimal growth under uncertainty. Journal of Economic Theory 11, 329-339.

Ng, S., Ruge-Murcia, F.J., 2000. Explaining the persistence of commodity prices. Computational Economics 16, 149-171.

Pindyck, R.S., Rotemburg, J.J., 1990. The excess co-movement of commodity prices. Economic Journal 100, 1173-1189.

Rincon-Zapatero, J.P., Rodriguez-Palmero, C., 2003. Existence and uniqueness of solutions to the Bellman equation in the unbounded case. Econometrica 71, 1519-1555.

Routledge, B.R., Seppi, D.J., Spatt, C.S., 2000. Equilibrium forward curves for commodities. The Journal of Finance 55, 1297-1338.

Samuelson, P.A., 1971. Stochastic speculative price. Proceedings of the National Academy of Science 68, 335-337.

Santos, M., Vigo, J.T., 1998. Analysis of a numerical dynamic programming algorithm applied to economic models. Econometrica 66, 409-426.

Schectman, J., Scheinkman, J.A., 1983. A simple competitive model with production and storage. Review of Economic Studies 50, $427-441$.

Stachurski, J., 2002. Stochastic optimal growth with unbounded shock. Journal of Economic Theory 106, 40-65.

Stokey, N.L., Lucas, R.E., Prescott, E.C., 1989. Recursive Methods in Economic Dynamics. Harvard University Press, MA.

Williams, J.C., Wright, B.D., 1991. Storage and Commodity Markets. Cambridge University Press, MA. 